\title{
Improved Training Sequence Channel Estimation Scheme in 16QAM MB-OFDM UWBoF System
}

\author{
Changqing Xiang $\mathbb{D}^{1},{ }^{1}$ Xi Yang, ${ }^{1}$ Xuan Zhou, ${ }^{1}$ Xiaojun $\mathrm{Zhu}^{2}{ }^{2}$ Jincan $\mathrm{Xu},{ }^{1}$ and Qiong $\mathrm{He}^{1}$ \\ ${ }^{1}$ College of Information Science and Engineering, Jishou University, Jishou, Hunan 416000, China \\ ${ }^{2}$ School of Software Engineering, Jiangxi University of Science and Technology, Nanchang, Jiangxi 330013, China
}

Correspondence should be addressed to Changqing Xiang; 531012578@qq.com

Received 11 July 2020; Accepted 1 October 2020; Published 14 October 2020

Academic Editor: Junmin Liu

Copyright (c) 2020 Changqing Xiang et al. This is an open access article distributed under the Creative Commons Attribution License, which permits unrestricted use, distribution, and reproduction in any medium, provided the original work is properly cited.

\begin{abstract}
In this paper, an improved channel estimation scheme based on time-domain orthogonal gray complementary training sequence (Golay TS) is proposed to resist subcarrier mutual beat interference in 16-quadrature amplitude modulation multiband orthogonal-frequency-division-multiplexing ultrawide band over fiber (16QAM MB-OFDM UWBoF) systems. The simulation results showed that the performance of system with the improved Golay TS channel estimation scheme was improved by about $1 \mathrm{~dB}$ compared with the traditional Golay TS channel estimation scheme, at a bit error rate (BER) of $3.8 \times 10^{-3}$ after $70 \mathrm{~km}$ standard single mode fiber (SSMF) transmission. At the same time, the synchronization and channel estimation performance of Golay TS and the selected frequency-domain orthogonal training sequences (TSs) were compared. The simulation results showed that the performance of system with the TSs channel estimation was improved by about $2.7 \mathrm{~dB}$ compared with the improved Golay TS channel estimation.
\end{abstract}

\section{Introduction}

As we all know, symbol timing synchronization is a key step that must be performed before signal demodulation at the receiving end of the signal. Therefore, the symbol timing synchronization step is very important [1-3]. If the symbol timing synchronization does not accurately find the start and end points of the symbol, it will lead to the possibility of phase noise. In addition, the intercarrier interference (ICI) $[4,5]$ and intersymbol interference (ISI) $[6,7]$ are also generated. However, in the course of MB-OFDM UWB signal transmission, fiber channel damage will inevitably be introduced. And the ISMI produced by photoelectric conversion (PD) will result in the transmission performance degradation obviously for the intensity modulation directdetection (IM/DD) structures [8]. In order to ensure that our original data can be recovered, the channel must be accurately estimated [9].

Recent studies have shown that the more commonly used channel estimation schemes are blind estimation and data-assisted estimation [10]. The advantage of blind estimation is that it does not need to introduce additional overhead, but its calculation complexity is very high, and the estimation effect is unsatisfying. It is not suitable for our MB-OFDM UWB signal. Data-assisted channel estimation schemes are mainly divided into two types: one is pilotassisted channel estimation scheme, and the other is training sequence-assisted channel estimation scheme [11]. For channel estimation algorithms, the least squares (LS) algorithm and the minimum mean square error (MMSE) algorithm are two common classic algorithms [12]. Generally, LS and MMSE are both channel estimation algorithms used in OFDM systems. However, the former does not consider the influence of noise, and the latter is very complex [13]. As for the MB-OFDM UWBoF system, whose channel estimation algorithm is used, it will depend on the specific situation $[14,15]$.

This paper proposed joint symbol synchronization and channel estimation scheme based on the same training sequence and improved the channel estimation scheme for 
ISMI. Simulation results showed that the improved Golay TS channel estimation scheme improved receiver sensitivity by about $1 \mathrm{~dB}$ compared with the previous scheme, but TSs played the best channel estimation performance.

$$
\begin{aligned}
X_{\mathrm{UWB}}(t) & =\operatorname{real}\left\{\sum_{k=1}^{N} C_{k} \exp (j 2 \pi k \Delta f t) \times \exp \left(j 2 \pi f_{\mathrm{IF}}[q(m)] t\right)\right\} \\
& =\operatorname{real}\left\{\left(I_{\mathrm{BB}}+j \cdot Q_{\mathrm{BB}}\right) \cdot\left[\cos \left(2 \pi f_{\mathrm{IF}}[q(m)] t\right)+j \cdot \sin \left(2 \pi f_{\mathrm{IF}}[q(m)] t\right)\right]\right\} \\
& =\operatorname{real}\left\{\begin{array}{c}
I_{\mathrm{BB}} \cdot \cos \left(2 \pi f_{\mathrm{IF}}[q(m)] t\right)-Q_{\mathrm{BB}} \cdot \sin \left(2 \pi f_{\mathrm{IF}}[q(m)] t\right)+ \\
j \cdot\left[I_{\mathrm{BB}} \cdot \sin \left(2 \pi f_{\mathrm{IF}}[q(m)] t\right)-Q_{\mathrm{BB}} \cdot \cos \left(2 \pi f_{\mathrm{IF}}[q(m)] t\right)\right]
\end{array}\right\} \\
& =I_{\mathrm{BB}} \cdot \cos \left(2 \pi f_{\mathrm{IF}}[q(m)] t\right)-Q_{\mathrm{BB}} \cdot \sin \left(2 \pi f_{\mathrm{IF}}[q(m)] t\right) \\
& =I_{\mathrm{IF}}-Q_{\mathrm{IF}},
\end{aligned}
$$

where $N=128$ represents the total number of subcarriers, $K$ represents the subcarrier sequence number, $q(m)$ represents the time frequency code (TFC) to which the symbol is mapped to the corresponding frequency according to the symbol, and $f_{\mathrm{IF}}[q(m)]$ represents the phase and frequency of the intermediate frequency (IF) carrier. $I_{\text {IF }}$ represents the real part of the generated IF MB-OFDM UWB signal, and $Q_{\mathrm{IF}}$ represents the imaginary part.

After the PD at the receiving end is photoelectrically converted and filtered, the resulting electrical MB-OFDM UWB signal can be further simplified as

$$
\begin{aligned}
I_{\mathrm{PD}-\mathrm{UWB}}(t)== & {\left[A_{0} \operatorname{Att}(L)\right]^{2} } \\
& +\left[A_{0} \gamma \operatorname{Att}(L)\left(I_{\mathrm{IF}}^{\prime}-\mathrm{Q}_{\mathrm{IF}}^{\prime}\right)\right]^{2} \\
& +A_{0}^{2} \gamma \operatorname{Att}^{2}(L)\left[\cos \left(\frac{\beta_{1}+\beta_{2}-2 \beta_{3}}{2} L\right)\right] \times\left(I_{\mathrm{IF}}^{\prime}-Q_{\mathrm{IF}}^{\prime}\right) \\
= & A_{0}^{2} \operatorname{Att}^{2}(L)\left[\gamma \cdot\left(I_{\mathrm{IF}}^{\prime}-\mathrm{Q}_{\mathrm{IF}}^{\prime}\right)+1\right] \\
& +\gamma^{2} A_{0}^{2} \mathrm{Att}^{2}(L) \cdot\left(I_{\mathrm{IF}}^{\prime}-Q_{\mathrm{IF}}^{\prime}\right)^{2} \\
& +\gamma A_{0}^{2} \operatorname{Att}^{2}(L) \cdot \cos \left(\frac{\beta_{1}+\beta_{2}-2 \beta_{3}}{2} L\right) \cdot\left(I_{\mathrm{IF}}^{\prime}-Q_{\mathrm{IF}}^{\prime}\right) .
\end{aligned}
$$

It can be seen from the above formula that the received signal is composed of three parts: $A_{0}^{2} \mathrm{Att}^{2}(L)\left[\gamma \cdot\left(I_{\mathrm{IF}}^{\prime}-\mathrm{Q}_{\mathrm{IF}}^{\prime}\right)+\right.$ $1]$ is the MB-OFDM UWB signal, $\gamma A_{0}^{2} \mathrm{Att}^{2}(L) \cdot \cos \left(\left(\beta_{1}+\right.\right.$ $\left.\left.\beta_{2}-2 \beta_{3} / 2\right) L\right) \cdot\left(I_{\mathrm{IF}}^{\prime}-Q_{\mathrm{IF}}^{\prime}\right)$ is the DC component, and $\gamma^{2} A_{0}^{2} \mathrm{Att}^{2}(L) \cdot\left(I_{\mathrm{IF}}^{\prime}-Q_{\mathrm{IF}}^{\prime}\right)^{2}$ is the ISMI. In addition, the MBOFDM UWB signal is also affected by the chromatic dispersion of the fiber during the transmission of the fiber, resulting in the MB-OFDM UWB symbol delay, resulting in frequency selective fading (FSF). Therefore, it is necessary to recover the original transmission data by means of symbol synchronization and channel estimation. This article used joint synchronization and channel estimation schemes based on Golay TS and TSs, respectively. The specific principles were described as follows.

2.1. The Technology for Channel Estimation Based on Golay TS. The Golay TS synchronization scheme adopted in this article refers to the method used by $\mathrm{He}[1]$. On this basis, the Golay TS used for synchronization continued to be used for channel estimation. In the direct-detection system, the traditional training sequence estimation generally adopted the LS algorithm, that is, the frequency domain was directly divided. For Golay TS with orthogonal time domain, all its 
subcarriers were used to estimate the channel response. The specific process was to transform Golay TS by fast Fourier transform (FFT) and transform time domain to frequency domain. Then, the Golay TS in the frequency domain of the receiving end was divided by the Golay TS in the frequency domain of the sending end. Therefore, the channel response function obtained by the channel estimation is defined as follows:

$$
H_{G}(k)=\frac{R_{T}(k)}{T(k)},
$$

where Golay TS in the frequency domain at the receiving end is expressed as $R_{T}(k)$ and the Golay TS in the frequency domain at the sending end is expressed as $T(k)$.

Considering that the MB-OFDM UWB signal at the receiving end can introduce the effect of ISMI interference after PD conversion and in the case of a small signal, other odd-numbered high-order interferences can be ignored [3]. And in the actual situation, the MZM may work in nonideal areas, so the temperature and humidity of the environment change may result in drifting of direct current offset, which may introduce ISMI interference. Therefore, when using channel estimation based on the Golay TS, the impact of ISMI interference introduced by the devices Mach-Zehnder modulator (MZM) and PD should be considered. Fortunately, the impact of ISMI interference only can fall on evennumbered subcarriers, and the data of odd-numbered subcarriers are not affected, so a simple and effective improvement scheme for traditional Golay TS channel estimation is used to estimate. The resulting channel response value is selected, and the channel response value on the odd subcarriers that are not affected by ISMI interference is retained. The channel response value on the even subcarrier affected by ISMI interference is deleted, and the new channel response value on the even subcarrier is determined by linear interpolation. The channel response value on the reserved odd subcarriers is obtained by interpolation. So, the improved channel response function based on Golay TS channel estimation can be expressed as

$$
H_{G}(k)_{\mathrm{opt}}=\left\{\begin{array}{l}
H_{\mathrm{odd}}(k)=\frac{R_{T}(k)}{T(k)}, \\
H_{\mathrm{even}}(k)=\frac{H_{\mathrm{odd}}(k+1)+H_{\mathrm{odd}}(k-1)}{2},
\end{array}\right.
$$

where ${ }_{R}^{T}(k)$ and $T(k)$, respectively, represent frequencydomain data received and transmitted on the corresponding $k$ subcarrier in the training sequence and $H_{\text {odd }}(k+1)$ and $H_{\text {odd }}(k-1)$, respectively, represent channel estimation values on odd subcarriers adjacent to the $k$ even subcarrier.

\subsection{The Technology for Channel Estimation Based on TSs.} For TSs, it is a training sequence constructed to resist ISMI interference that is introduced by the device characteristics of MZM and PD. Its special structure determined the effect of suppressing ISMI interference. Given that the ISMI interference can be introduced after passing through the PD which only fell on even-numbered subcarriers, the channel obtained from the training sequence may estimate that its odd-numbered subcarriers will not be affected by ISMI interference. Therefore, the channel estimation based on the TSs can be easily obtained.

After symbol synchronization [16], the frequency-domain data of TSs can be obtained after FFT demodulation. Since the symbol modulation of BPSK is only performed on odd subcarriers, we can directly use the single-tap frequency-domain estimation scheme to obtain the corresponding channel response, whose function on its odd subcarriers can be defined as follows:

$$
H_{\text {odd }}(k)=\frac{R_{T}(k)}{T(k)},
$$

wherek denotes odd numbers and ${ }_{R}^{T}(k)$ and $T(k)$, respectively, represent frequency-domain data received and transmitted on the corresponding $k$ subcarrier in the training sequence. After obtaining $H_{\text {odd }}(k)$, the corresponding channel response function on its even subcarrier can be obtained through linear interpolation, and it is expressed as

$$
H_{\text {even }}(k)=\frac{H_{\text {odd }}(k-1)+H_{\text {odd }}(k+1)}{2},
$$

where $k$ denote even numbers and $H_{\text {odd }}(k+1)$ and $H_{\text {odd }}(k-1)$, respectively, represent channel estimation values on odd subcarriers adjacent to the $k$ even subcarrier.

At that time, the channel response function based on TSs channel estimation can be expressed as

$$
H_{\mathrm{TSs}}(k)=\left(\begin{array}{l}
H_{\text {odd }}(k)=\frac{R_{T}(k)}{T(k)}, \\
H_{\text {even }}(k)=\frac{H_{\text {odd }}(k+1)+H_{\text {odd }}(k-1)}{2} .
\end{array}\right)
$$

In general, the training sequence is accompanied by many MB-OFDM UWB data symbols, and the system overhead brought by training sequence can almost be negligible. Therefore, the scheme of joint synchronization and channel estimation based on a single training sequence has the advantages of low complexity and high accuracy, so it is suitable for such quasistatic channels of optical fiber systems.

\section{Simulation System and Result Analysis}

The simulation setup of the MB-OFDM UWBoF transmission system based on the joint synchronization and channel estimation scheme of a single training sequence is shown in Figure 1. At the signal transmitter, the generated pseudorandom binary sequence (PRBS) was bit-interleaved and mapped to generate 16QAM symbols, and pilot frequency was inserted. Scheme (1): three Golay TSs were added to the three subbands to estimate the channels of the three subbands, and the Golay TS on the first subband was 


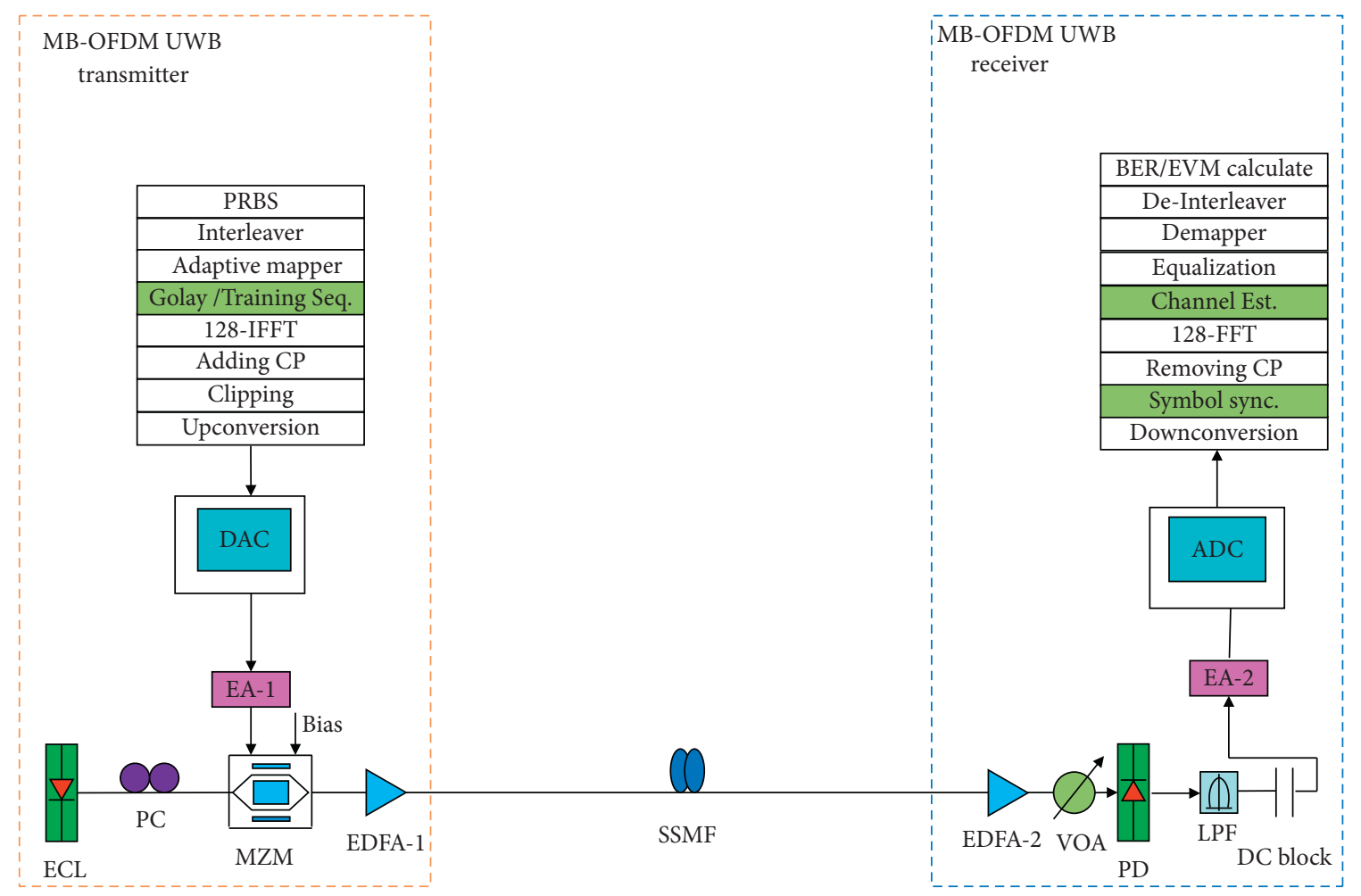

FIGURE 1: Simulation diagram of the MB-OFDM UWBoF transmission system based on a single training sequence scheme.

also used for symbol synchronization; scheme (2): three TSs were added to the three subbands to estimate the channels of the three subbands, and the TSs on the first subband were also used for symbol synchronization. Subsequently, 128point IFFT was used to realize the OFDM modulation of the subcarrier, a cyclic prefix was added, and then the OFDM signal was clipped. After digital up-conversion, the input window of MATLAB was imported into OptiSystem simulation software for digital-to-analog conversion to analog signal output for fiber transmission. Its basic parameters are listed in Table 1.

At the receiver, the captured signal was exported through the MATLAB output window for offline DSP processing. MB-OFDM UWB digital signals were digitally downconverted to baseband signals. Training sequences were used to achieve symbol synchronization, CP data removal, and OFDM signal demodulation. After that, according to the scenario of (1), the channel responses on the corresponding three subbands were estimated by extracting the three used Golay TSs, respectively; according to the scenario of (2), the estimated and compensated channel responses on the three sub-bands separately were obtained by extracting the three TSs. Subsequently, after performing channel equalization on each data subcarrier, the demodulated output signal of OFDM was sent to the de-interleaver and demapped into the original transmission bit stream. And the recovered data were placed in the BER/error vector magnitude (EVM) calculation module to calculate the bit error rate and EVM value.

We compared the synchronization performance of Golay TS and TSs. As shown in Figure 2(a), although the
Golay TS peak was very sharp without sidelobes, the complexity of its synchronization algorithm is very high due to its special structure. Relatively speaking, the synchronization algorithm of TSs was very simplified. Therefore, the calculation complexity was greatly reduced, and it can also be accurately synchronized. The timing measurement of TSs symbol was shown in Figure 2(b). The system for direct detection required low complexity, low cost, and simple implementation. Therefore, TSs were more competitive under the condition of accurate synchronization.

In terms of channel estimation, for the 16QAM MBOFDM UWBoF system, the BER performance of the channel estimation based on three training sequences was simulated after $70 \mathrm{~km}$ SSMF transmission. It can be seen from Figure 3 that the system performance estimated based on the Golay TS channel estimation scheme was more general. The reason might be that Golay TS is unable to eliminate the ISMI interference of its even subcarriers, which may lead to performance degradation. For ISMI interference, we improved the channel estimation of Golay TS, using only odd subcarrier data to estimate the channel and then interpolating to obtain the channel estimation scheme for all subcarriers. As the ISMI interference was suppressed, the performance of channel estimation was significantly improved, which may ascribe to the fact that Golay TS is a timedomain orthogonal training sequence but TSs are frequencydomain orthogonal training sequences. It is well known that frequency-domain orthogonal training sequences were superior to time-domain orthogonal training sequences in channel estimation performance. Therefore, the system performance estimated based on the TSs channel estimation 
TABLE 1: The main parameters of the transceiver based on a single training sequence scheme.

\begin{tabular}{lc}
\hline Parameter name & Parameter value \\
\hline Single OFDM symbol duration & $312.5 \mathrm{~ns}$ \\
FFT/IFFT size/number of subcarriers & 128 \\
CP and ZPS & $37(=16+21)$ \\
Data/pilot/null subcarrier/guard interval & $100 / 12 / 6 / 10$ \\
Bandwidth (relative to one of the sub-bands) & $528 \mathrm{MHz}$ \\
Number of TS/OFDM symbols & $3 / 312$ \\
DAC and ADC sampling rate & $10.561 \mathrm{GSps}$ and $20 \mathrm{GSps}$ \\
Modulation format & $16 \mathrm{QAM}$ \\
The total number of bits per OFDM symbol & $16 \mathrm{AM}: 100 * 4=400 \mathrm{bits}$ \\
Original signal bit rate & $16 \mathrm{QAM}: \log 2(16) *(1 / 312.5) * 100=1.28 \mathrm{Gbps}$ \\
Signal net bit rate & $400 * 312 /(315 * 165 * 20 / 10.561)=1.268 \mathrm{Gbps}$ \\
Optical spectrum efficiency & $1.268 /(100 / 128 * 0.528)=3.074 \mathrm{bit} / \mathrm{s} / \mathrm{Hz}$ \\
\hline
\end{tabular}

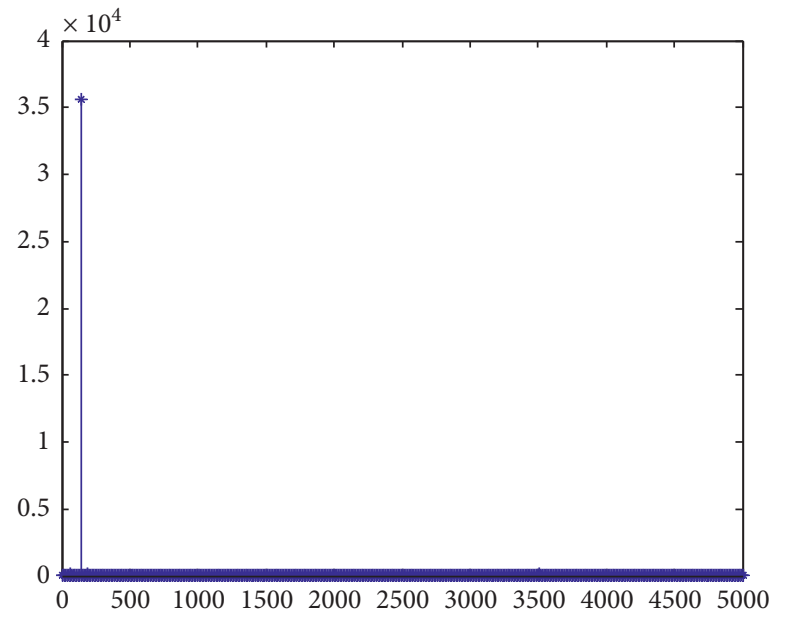

(a)

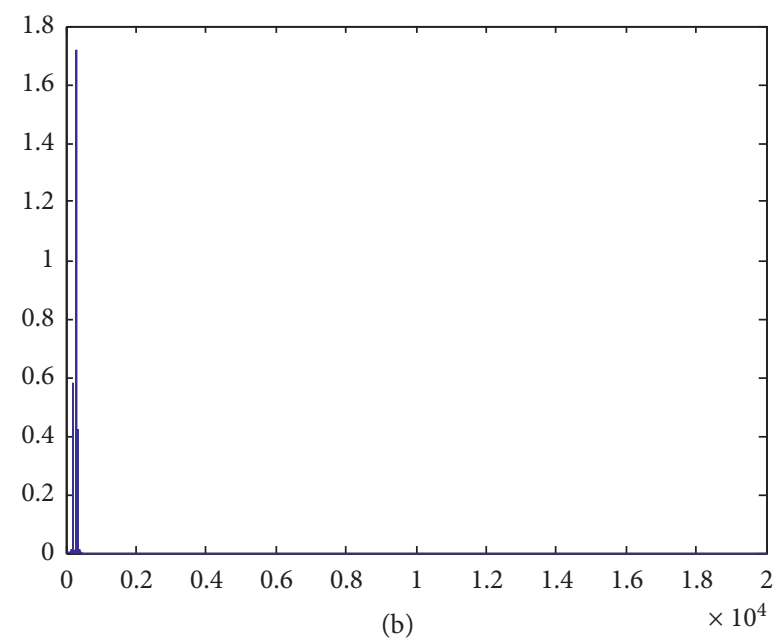

(b)

FIgURE 2: (a) MB-OFDM UWB signal frame timing synchronization metrics based on Golay TS scheme. (b) TS-based timing synchronization metrics for MB-OFDM UWB signal frames.

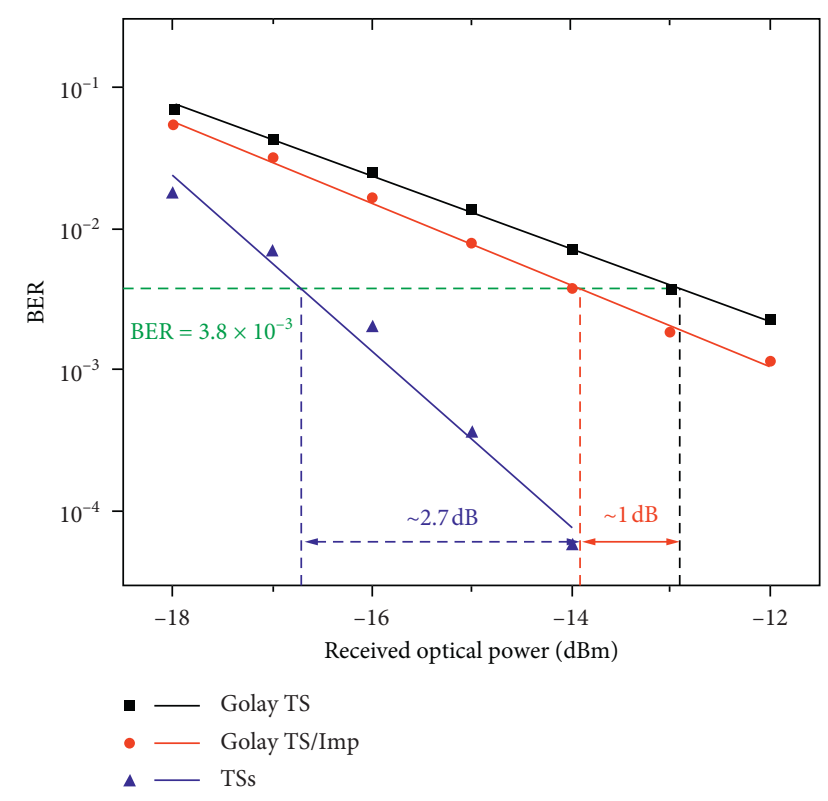

FIGURE 3: BER performance based on channel estimation of three training sequences. scheme was the best. Specifically, when the BER was $3.8 \times 10^{-3}$, the receiver sensitivity of the signal was improved by about $1 \mathrm{~dB}$ compared with the original Golay TS auxiliary channel estimation to scheme by using the improved Golay TS auxiliary channel estimation scheme in the 16QAM MBOFDM UWBoF system. Compared with the improved Golay TS auxiliary channel estimation scheme, the receiver sensitivity of the 16QAM MB-OFDM UWB signal was improved by about $2.7 \mathrm{~dB}$ when using the TSs auxiliary channel estimation scheme.

After the simulation through $70 \mathrm{~km}$ SSMF transmission, the 16QAM MB-OFDM UWB signal was based on the constellation diagram of the three training sequence channel estimates when the received optical power was $-16 \mathrm{dBm}$. Figure 4 (a) shows the traditional constellation. The constellation diagram obtained after the Golay TS auxiliary channel estimation scheme was estimated. Figure 4(b) presents the constellation diagram obtained from the estimation based on the improved Golay TS auxiliary channel estimation scheme. Figure 4(c) demonstrates the constellation based on the TSs. Compared with the traditional Golay TS auxiliary 


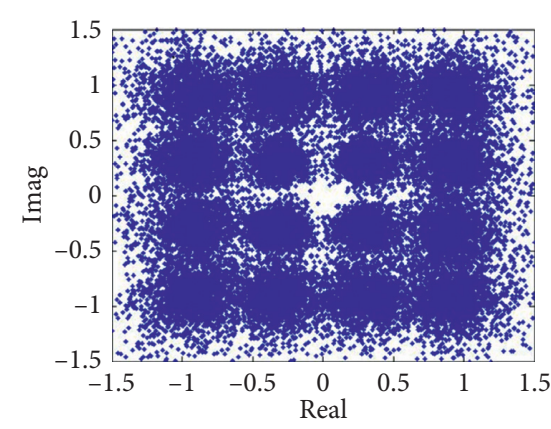

(a)

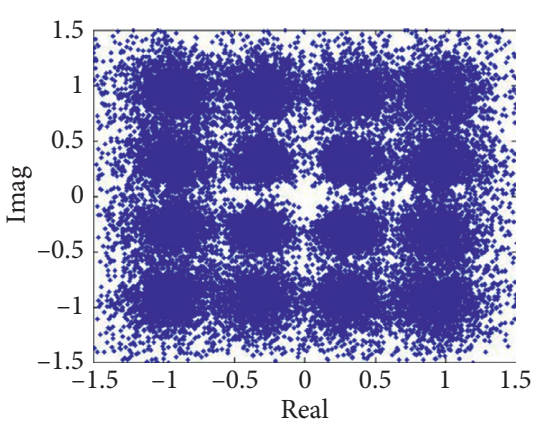

(b)

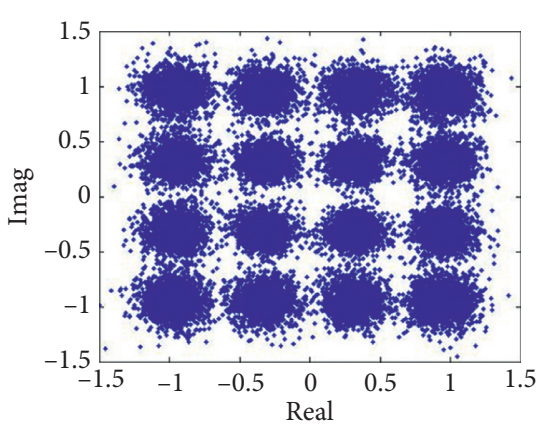

(c)

Figure 4: Constellation diagram based on channel estimation of three training sequences.

channel estimation scheme, the constellation diagram based on the improved Golay TS auxiliary channel estimation scheme was more convergent. Meantime, the distribution of the constellation diagram was more uniform and clearer. The performance of the constellation obtained from estimation based on TSs auxiliary channel estimation scheme was the best when comparing with the other two schemes.

\section{Conclusion}

The channel estimation scheme proposed in this paper adopted only odd-numbered subcarrier data. Then, interpolates were used to obtain the channel estimation scheme for all subcarriers. Since the ISMI interference was suppressed, the performance of channel estimation was significantly improved. The simulation results showed that the improved scheme could increase the receiver sensitivity of the signal by around $1 \mathrm{~dB}$ when using Golay TS-assisted channel estimation. The receiver sensitivity of TSs channel estimation was improved by about $2.7 \mathrm{~dB}$ compared with the improved Golay TS channel estimation.

\section{Data Availability}

No data used to support the findings of the study.

\section{Conflicts of Interest}

The authors declare that they have no conflicts of interest.

\section{Acknowledgments}

The authors really appreciate the support by the National Natural Science Foundation of China (61861019), the Natural Science Foundation of Hunan Province of China (2019JJ50483), the project of Hunan Provincial Department of Education (18B316 and 18C0588), and the Scientific Research Foundation of Hunan Province Higher Education Key Laboratory of Modeling and Monitoring on the NearEarth Electromagnetic Environments (N201901).

\section{References}

[1] V. Kishore, V. S. Prasad, and V. V. Mani, "A blind timing synchronization algorithm for DCO-OFDM VLC systems," IEEE Photonics Technology Letters, vol. 32, no. 17, pp. 11211124, 2020.

[2] Bruno and X. Zhai, "Performance of coarse timing synchronization in orthogonal frequency division multiplexing system," Journal of Physics Conference Series, vol. 960, 2018.

[3] Z. Hu, D. He, and Z. Xie, "OFDM symbol timing synchronization algorithm for PLC," Electric Power Automation Equipment, vol. 39, no. 5, pp. 144-150, 2019.

[4] M. Simko, C. Mehlfuhrer, T. Zemen, and M. Rupp, "Intercarrier interference estimation in MIMO OFDM systems with arbitrary pilot structure," in Proceedings of the Vehicular Technology Conference, pp. 1-5, Yokohama, Japan, May 2011.

[5] S. Jasdeep and A. Komal, "Inter carrier interference removal in MIMO-OFDM system," IOSR Journal of Electronics and Communication Engineering, vol. 9, no. 2, pp. 87-91, 2014.

[6] C. Gong and Z. Xu, "Channel estimation and signal detection for optical wireless scattering communication with intersymbol interference," IEEE Transactions on Wireless Communications, vol. 14, no. 10, pp. 5326-5337, 2015.

[7] J. Remple and I. Galton, "The effects of inter-symbol interference in dynamic element matching DACs," IEEE Transactions on Circuits and Systems I: Regular Papers, vol. 64, no. 1, pp. 14-23, 2017.

[8] Z. Cao, G. Wen, F. Li, Q. Shu, J. Yu, and L. Chen, "Unbalanced impairments compensation for low cost direct detection OFDM-PON systems," Optics Communications, vol. 310, pp. 35-41, 2014.

[9] M. Chen, G. Liu, L. Zhang et al., "Hardware-efficient implementation and experimental demonstration of Hermitian-symmetric IFFT for optical DMT transmitter," Optics Express, vol. 27, no. 21, pp. 29817-29828, 2019.

[10] B. Yang, Z. Cao, and K. B. Letaief, "Analysis of low-complexity windowed DFT-based MMSE channel estimator for OFDM systems," IEEE Transactions on Communications, vol. 49, no. 11, pp. 1977-1987, 2001.

[11] S. Coleri, M. Ergen, A. Puri, and A. Bahai, "Channel estimation techniques based on pilot arrangement in OFDM systems," IEEE Transactions on Broadcasting, vol. 48, no. 3, pp. 223-229, 2002.

[12] J. S. Parmar and G. Gupta, "DFT-based channel estimation for OFDM system and comparison with LS and MMSE over Rayleigh and Rician fading channel," International Journal of Engineering Trends \& Technology, vol. 4, no. 9, 2013. 
[13] Y. Liu, Z. Tan, H. Hu, L. J. Cimini, and G. Y. Li, "Channel estimation for OFDM," IEEE Communications Surveys \& Tutorials, vol. 16, no. 4, pp. 1891-1908, 2014.

[14] J. He, C. Xiang, F. Long, and Z. Chen, "Experimental research of UWB over fiber system employing 128-QAM and ISFAoptimized scheme," Optical Fiber Technology, vol. 42, pp. 151-155, 2018.

[15] J. He, C. Xiang, F. Long, K. Wu, and L. Chen, "Pre-compensation combined with TS-aided and ISFA-enhanced scheme for UWB system," Optics Communications, vol. 396, pp. 150-155, 2017.

[16] M. Chen, J. He, and L. Chen, "Real-time optical OFDM longreach PON system over $100 \mathrm{~km}$ SSMF using a directly modulated DFB laser," Journal of Optical Communications and Networking, vol. 6, no. 1, pp. 18-25, 2014. 